\title{
A Comment on Craig et al.: "Retigabine for the Adjunctive Treatment of Adults with Partial-Onset Seizures in Epilepsy with and without Secondary Generalization: A NICE Single Technology Appraisal"”
}

\author{
Przemystaw Holko $\cdot$ Pawel Kawalec
}

Published online: 29 March 2013

(C) The Author(s) 2013. This article is published with open access at Springerlink.com

Dear Editor,

In their publication of the National Institute for Health and Clinical Excellence single technology appraisal of retigabine, Craig et al. [1] report the results of the network comparison of retigabine and other anti-epileptic treatments.

We find this to be an interesting article as well as the entire series of single technology appraisal articles published in PharmacoEconomics, considering that single appraisals are common in the Health Technology Assessment process for drug reimbursement in Poland.

We agree with Craig et al.'s conclusions [1], although we believe that the relationship between the dose of retigabine and the effect should be more intensively examined, especially in comparison with other treatments.

The available clinical data (cf. Craig et al. for details [1, 2]) allows for investigation of dose-response effects. The conclusions from a dose-dependent model may be more useful for clinicians who are only at the beginning of their experience with a new anti-epileptic drug, such as retigabine. Presentation of retigabine dose corresponding to the effectiveness or safety profile of other interventions would allow for better planning of the treatment, such as in determining the magnitude of the retigabine dose increase in the titration phase.

Using the clinical data for the intent-to-treat population (titration and maintenance phase combined), Craig et al. [1]

\section{P. Holko}

Centrum HTA, Krakow, Poland

P. Kawalec $(\bowtie)$

Drug Management Department, Institute of Public Health, Faculty of Health Sciences, Jagiellonian University, Grzegorzecka 20, 31-531 Krakow, Poland

e-mail: pawel.kawalec@uj.edu.pl reported low heterogeneity of the effect after pooling all doses of retigabine. The lack of dose dependence is, however, counter-intuitive and, acknowledging the homogeneity of the trials of retigabine, meta-regression should be carried out, even with the assumption that the only predictor of the effect is the actual dose of retigabine (due to the insufficient number of trials for conducting a more complex model [3]).

For retigabine, lacosamide and eslicarbazepine-treatments with available clinical data allowing plausible analysis of the dose-effect relationship-we conducted a meta-analysis and meta-regression. The results of our calculations are shown in Table 1.

The meta-regression results are consistent with the results of a meta-analysis of individual doses, are associated with lower rates of heterogeneity (Cochrane Q test; data not shown), and are intuitive (increasing the dose we expect a higher effect, particularly in a trial with a forced titration phase).

As an example, we found that for each daily dose of a 200-mg increase of retigabine, eslicarbazepine and lacosamide, the odds for the response (with at least a $50 \%$ reduction in the frequency of seizures) increases were: odds ratio (OR) 1.24 (95\% credible interval [CrI]: 1.16-1.33), OR 1.22 (95\% CrI: 1.13-1.33) and OR 1.41 (95\% CrI: 1.22-1.64), respectively, with a significant dose-dependent effect across all treatments $(p<0.001)$.

Likewise we found a highly significant dose-dependent effect across all treatments for the rate of adverse events leading to discontinuation $(p<0.0001)$, yet a nonsignificant (retigabine, lacosamide) or small (eslicarbazepine; $p=0.0276)$ impact of dose variation on the rate of seizure freedom (most likely due to limitations of rare event analysis).

Expanding the meta-regression model with an indirect comparison, it could be estimated that in order to obtain the 
Table 1 Effectiveness of retigabine, lacosamide and eslicarbazepine in comparison with placebo: odds ratio with $95 \%$ CI (meta-analysis results) or $95 \% \mathrm{CrI}$ (meta-regression results) in brackets (ITT population)

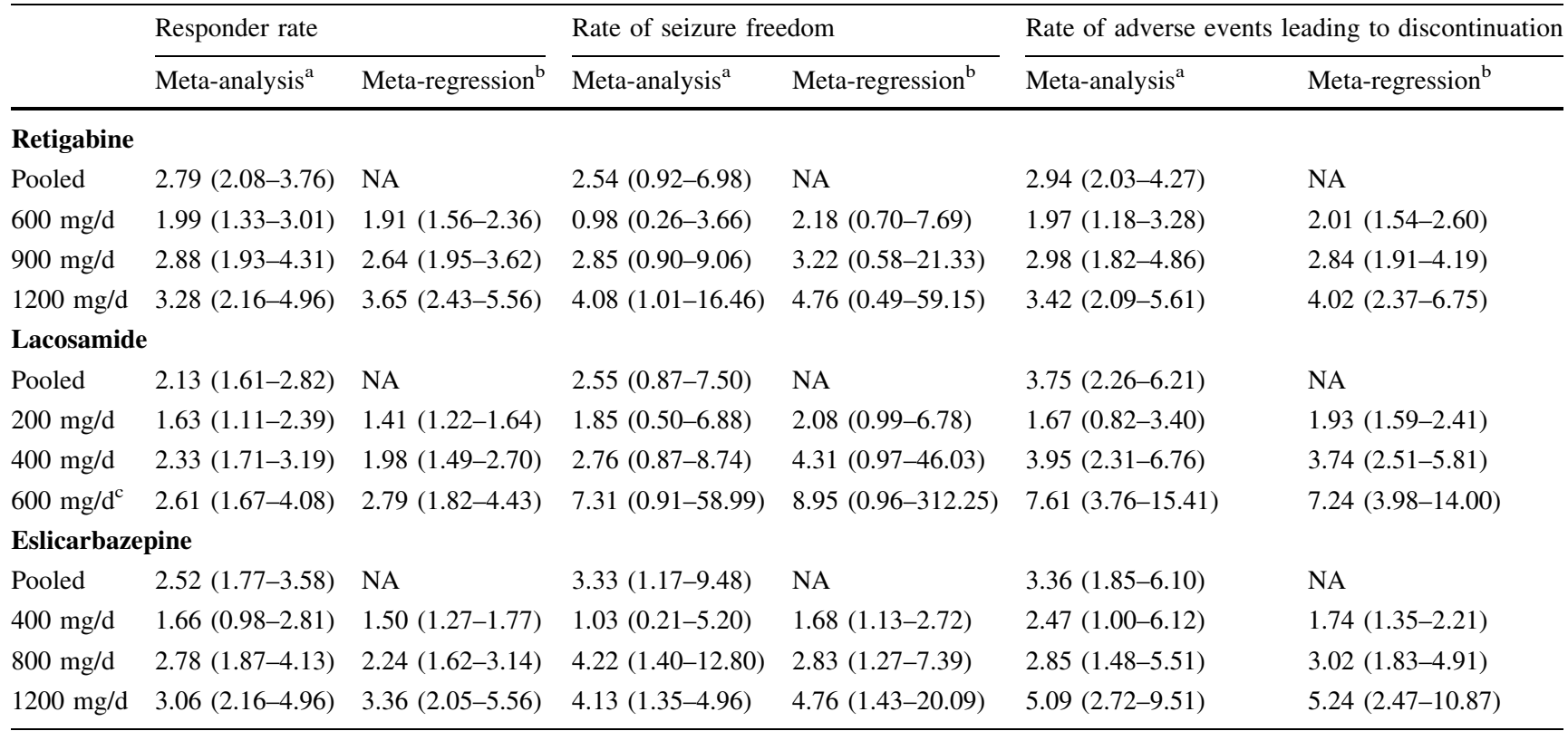

$O R$ odds ratio, $C I$ confidence interval, $C r I$ credible interval, $N A$ not applicable, ITT intent-to-treat

${ }^{a}$ Mantel-Haenszel fixed-effects model included studies identified by Craig et al. [1, 2]

b Bayes random effect logistic model [3] with the dose of each drug as the only independent variable

c Dosage not recommended [4]

Table 2 Dose of lacosamide and eslicarbazepine corresponding to the effectiveness or safety profile of retigabine at a dose of $600 \mathrm{mg} / \mathrm{d}$

\begin{tabular}{lll}
\hline Outcome & Dose of lacosamide & Dose of eslicarbazepine \\
\hline Responder rate & $411 \mathrm{mg} / \mathrm{d}\left(95 \% \mathrm{CrI}: 247-909^{\mathrm{a}}\right)$ & $632 \mathrm{mg} / \mathrm{d}(95 \% \mathrm{CrI}: 395-1091)$ \\
Rate of seizure freedom & $511 \mathrm{mg} / \mathrm{d}\left(-^{\mathrm{b}}\right)$ & $220 \mathrm{mg} / \mathrm{d}\left(-^{\mathrm{b}}\right)$ \\
Rate of adverse events leading to discontinuation & $216 \mathrm{mg} / \mathrm{d}(95 \%$ CrI: $75-568)$ & $490 \mathrm{mg} / \mathrm{d}\left(95 \% \mathrm{CrI}: 174-1383^{\mathrm{a}}\right)$ \\
\hline
\end{tabular}

CrI credible interval

${ }^{\text {a }}$ Higher than the maximum recommended dose $[4,5]$

${ }^{\mathrm{b}}$ Uncertainty range greatly exceeding the dosage in clinical practice and/or being counter-intuitive (negative value)

same responder rate as for retigabine at a dose of $600 \mathrm{mg} /$ $\mathrm{d}$, eslicarbazepine should be given at a dose of $632 \mathrm{mg} / \mathrm{d}$ (95 \% CrI: 395-1091) and lacosamide at a dose of $411 \mathrm{mg} /$ d (95 \% CrI: 247-909). Since the equivalent dosage could be quite different with different outcome measures (Table 2), the most suitable outcome selection should be consulted with practitioners or the competition/weighing of the different events should be included.

Furthermore, the proposed approach could be helpful in identifying the potential differences in the safety and effectiveness of a new anti-epileptic drug. In our example, the point estimate of equivalent doses might indicate that retigabine could be associated with a lower risk of discontinuation due to adverse events and with a similar response rate when compared with lacosamide or eslicarbazepine (with an equal safety profile achieved at a lower dose than the equal rate of response). However, this conclusion would need further examination.

In conclusion, a simplified solution was shown to be only one of the possible approaches to the investigation of dose-response effects. We are aware of the limitations of the proposed model and indicate that a more accurate approach would be required; i.e. the inclusion of all treatments and all events (in particular the response to the treatment and withdrawal due to adverse events or serious adverse events), which additionally requires dealing with methodological issues associated with the relationship of the safety profile to the effectiveness of anti-epileptic treatments. We believe, however, that even with certain limitations a similar approach would be more informative 
to the public and practitioners than the approach adopted by Craig et al. [1].

Acknowledgments The authors have no conflicts of interest to disclose in relation to the content of this letter.

Open Access This article is distributed under the terms of the Creative Commons Attribution Noncommercial License which permits any noncommercial use, distribution, and reproduction in any medium, provided the original author(s) and the source are credited.

\section{References}

1. Craig D, Rice S, Paton F, Fox D, Woolacott N. Retigabine for the adjunctive treatment of adults with partial-onset seizures in epilepsy with and without secondary generalization: a NICE single technology appraisal. Pharmacoeconomics. 2013;31(2): 101-10.

2. Craig D, Rice S, Paton F, Fox D, Woolacott N. Retigabine for the adjunctive treatment of adults with partial onset seizures in epilepsy with and without secondary generalisation: a single technology appraisal. York: Centre for Reviews and Dissemination and Centre for Health Economics, University of York; 2011 [online]. http://www.hta.ac.uk/erg/reports/2478.pdf. Accessed 26 Jan 2013.

3. Thompson SG, Sharp SJ. Explaining heterogeneity in meta-analysis: a comparison of methods. Stat Med. 1999;18(20):2693-708.

4. European Medicines Agency. Vimpat $50 \mathrm{mg}$ : summary of product characteristics [online]. http://www.emea.europa.eu/docs/en_GB/ document_library/EPAR_-_Product_Information/human/000863/ WC500050338.pdf. Accessed 26 Jan 2013.

5. European Medicines Agency. Zebinix $200 \mathrm{mg}$ : summary of product characteristics [online]. http://www.emea.europa.eu/docs/ en_GB/document_library/EPAR_-_Product_Information/human/ 000988/WC500047225.pdf. Accessed 26 Jan 2013. 